J. EDUCATIONAL COMPUTING RESEARCH, Vol. 26(2) 191-201, 2002

\title{
EFFECTS OF WHITE SPACE IN LEARNING VIA THE WEB
}

\author{
AMY C. BRADSHAW \\ University of Oklahoma \\ ABBAS JOHARI \\ Cameron University
}

\begin{abstract}
This study measured the effect of specific white space features on learning from instructional Web materials. The study also measured learners' beliefs regarding Web-based instruction. Prior research indicated that small changes in the handling of presentation elements can affect learning. Achievement results from this study indicated that in on-line materials, when content and overall structure are sound, minor differences regarding table borders and vertical spacing in text do not hinder learning. Beliefs regarding Web-based instruction and instructors who use it did not differ significantly between treatment groups. Implications of the study and cautions regarding generalizing from the results are discussed.
\end{abstract}

Web-based and Web-supported instructional development is increasing rapidly. The Web promises access to immense amounts of information quickly and easily, and offers exciting options to teachers and learners, both at a distance and in conventional settings. Instructional Web sites are available on nearly any topic for any age. Unfortunately, many instructional sites include elements of visual, structural, or content design that can hinder learning. Sites that are poorly designed, visually or structurally, may diminish or negate the Web's potential benefits. Previous research in the realm of learning from computer mediated visual presentations indicates that even where structure and content are sound, decisions regarding visual design can affect subjects' ability to learn from the material presented [1]. Two constructs related to this framework are Presentation Interference and Cognitive Overhead. Presentation Interference has been defined as 
any presentation-related factor that distracts the learner from the message content [2]. Examples include inappropriate color choices, inconsistent or incompatible screen transitions, inconsistent navigation devices, spelling and grammatical errors, etc. Cognitive Overhead [3], refers to the negative influence distracting factors such as presentation interference have on learning. The premise is that increased cognitive overhead results in increased challenge and difficulty for learners in focusing on and processing vital content. Distractions caused by relatively insignificant elements of an instructional presentation, for example, inconsistent placement of navigation icons, make learning more difficult because learners must reorient themselves to structural and screen elements when they should be attending to content.

As with all other media, Web developers need to know that cluttered documents communicate less [4, 5] and design elements should always be planned [6]. Rich research has been conducted in the area of learning from instructional text with regard to legibility. For example, to help learners understand the structure of the content, developers should use white space differently around various levels of headings [7-9]. To differentiate paragraphs, vertical space is more effective than indention [8]. White space is an important component of readability that is generally considered to make text easier to read $[10,11]$.

White space is any part of the page or screen that does not have text or graphical elements [12]. White space has at least six forms: 1) the margins, 2) the area surrounding the headings and between headings and corresponding paragraphs, 3) the space at the end of lines, 4) the leading, 5) the tracking, and 6) the space around images and graphic elements. Appropriate use of white space forms provides for powerful visual design [9].

Among the benefits of white space are the following: appropriate use of white space facilitates contrast [13], simplicity, and balance in a document. White space can create tension between two design elements [14]. Empty space provides resting points within a page that may facilitate deeper processing. In printed instructional text, developers frequently have used boxes to extend the reader's comprehension of the main ideas, but research has not reported on its effectiveness [15]. Applied in isolation, generic guidelines can be of limited usefulness [2]. As with all other design choices, context, including topic, audience, setting, and medium, is important to determining appropriateness. Above all, the appropriateness of white space must be considered holistically, in relation to all other design choices and elements on or within the same page or document. As a design element [16, 17], its use should be as thoughtful as any other.

Recent research in this area utilizing modern media is minimal and many text-specific guidelines lack true experimental and treatment designs, particularly with comparisons of actual practice versus professional standards (as opposed to two treatments that are merely different from each other). There is a need to re-examine legibility of text, specifically spatial arrangement in the context of 
instructional Web pages. Although both deal with large amounts of text, the context of the Web is very different from the context of a printed page, or even from Computer Based Instruction (CBI) screens. For example, in a printed page white space generally has a positive connotation, although its use may be limited by the need to conform to specific numbers of pages. In a CBI program, the amount of text on a single screen can be greatly reduced and the amount of white space increased because it costs virtually nothing to add additional screens, thereby making the information on any single screen appear easier to access. However, what would appear to be an optimal amount of white space in a CBI program would, in the context of a Web page, be considered wasteful and bad practice because it would create the need for scrolling to get to all the information. Nielson discusses the need to limit the amount of white space to just what is necessary for ease of usability [18]. Further, the presence of hyperlinks in Web pages allows users to think about, use, and pursue information differently than with a static printed page. This may result in differences regarding learners' perceptions, motivation, and effort when using the Web, thereby affecting their abilities to learn using Web-based text. The topic is important for developers interested in transferring curricula to the Web and in understanding how to develop on-line multimedia instruction that is visually sound and that should promote better learning.

Research in a context of computer generated presentations has indicated that small changes with regard to presentation factors can affect achievement and beliefs [1]. In that study, an instructional presentation that was intentionally presentation interference free resulted in higher achievement scores than did presentations with interference. Further, although subjects who learned from visually well designed presentations and those who learned from presentations containing presentation interference both reported strong beliefs regarding the beneficial nature of computer generated presentations, including that they believed they had "learned a lot" from the treatment, the treatment groups viewing presentations containing interference learned significantly less as measured by the achievement test.

The present study examined similar hypotheses in the context of information via the Web. The purpose of this study was to determine whether small changes in white space related factors (e.g., structural white space, visible table borders) affected learning from Web-based instructional materials as measured by achievement and belief. In particular, it measures effects of white space in an intentionally presentation interference-free instructional Web presentation vs. similar lessons with inappropriate handling of white space.

Hypothesis 1: Subjects receiving treatment one, with white space and no visible table borders, would score higher on the achievement test than would subjects in treatments two or three, without white space structural cues and with visible table borders. 
Hypothesis 2: Subjects viewing treatment one, the interference free presentation, would report more positive beliefs regarding 1) the treatment site, 2) learning from the Web in general, and 3) instructors who include Internet use in their classes, than would those viewing presentations containing presentation interference.

\section{METHOD}

\section{Subjects}

Subjects were 47 undergraduates enrolled in first-year multimedia design and production courses at a small, public, four-year university in the southwestern United States during the spring 2000 semester. Participation was voluntary. Subjects were randomly assigned to three treatment groups. Following the treatments, subjects completed an immediate achievement test and belief questionnaire.

\section{Materials}

\section{Treatments}

A Web site presentation was developed and presented via Windows-based computers with high resolution monitors and Netscape Communicator. The treatments presented types, incidence, and identification of skin cancer, and steps to reduce risk (adapted from [1]). The "control" version was designed according to known screen design research findings and prescriptive guidelines, was free of intentional interference, and included a great deal of white space, without visible tables, lines, or borders. The second version of the presentation was identical to the control version with one exception, the borders of tables used to organize the information were visible. The result was borders very close to the text without the "pixel padding" that would allow space between border and text. The third version was identical to the second with one exception: the structural white space was eliminated, resulting in more condensed text and, in some instances, text no longer directly beside related graphics. Across treatment groups, all factors remained constant except the handling of white space and visible table borders on the premise that small changes in a page may affect learning. This premise was based on results from previous research in which small changes in screen design and presentation factors between treatments did result in significant differences across treatment groups.

Instrument

A two-part instrument was used to collect data. The first part was a 25 -item "short answer" and "fill in the blank" achievement test regarding the information 
presented. The second portion was a series of 12 seven-point bipolar probability items regarding subjects beliefs. Questions 1-5 of the belief questionnaire referred to students' beliefs regarding the credibility of the site and how much they learned from it. Questions 7-9 referred to subjects' beliefs regarding Web-based information and instructors who use it. Question 10 referred to the subjects' own experiences with Web development, and questions 6, 11-12 referred to subjects' beliefs about their behavior before and after visiting the site.

Content validity was assured in the following manner: Both test and treatments were developed using information and photographs distributed by the American Cancer Society and the Mayo Clinic. Posttest items were parallel to the instruction. Both the test and the content were evaluated by the researchers, two software development teachers, and one corporate instructional designer. Using a split half procedure, the reliability coefficient for the posttest was 0.82 .

\section{Procedures}

An experimental posttest-only control group design was used. Randomization of subjects into treatment groups was used to assure absence of bias. The independent variable was treatment. The dependent variables were 1) the percentage of correct responses to the follow-up test and 2) responses to the belief questionnaire.

Data were collected during a four-week period to increase the number of participants and to more closely mimic students' uses of the Internet for learning. Subjects chose their own participation times from a variety of times offered, without the pressure of a contrived classroom atmosphere, and were randomly assigned to one of three treatment groups. Study participants learned from a Web-based lesson, then completed an achievement test and belief questionnaire. The treatment took subjects about 15 minutes to complete. All subjects completed the questionnaires immediately following the treatment and were allowed as much time to complete the posttest and questionnaire as they desired. Completing the questionnaire required 10-20 minutes.

\section{Data Analysis}

The number of points correctly answered on the posttest was converted to a percentage of the items possible. Data from the posttest were analyzed using one-way analysis of variance (ANOVA). Significance was set at the .05 level. SPSS was used to analyze the data. Responses to items on the belief questionnaire also were analyzed using ANOVA, with significance set at the .05 level.

\section{RESULTS}

The study considered two hypotheses. The first predicted that subjects receiving treatment one, with white space and no table borders, would score higher on the achievement test and that subjects in treatment three, without white space 
structural cues and with visible table borders, would score lower than subjects in both treatments one and two. The second hypothesis examined responses to a 10-item belief questionnaire regarding the treatment and the use of the Web for instruction in general. Analysis of variance was used to analyze data regarding each of the questions.

\section{Achievement}

All subjects completed a 25 -item achievement test immediately following the treatment. Means achievement scores for Treatments groups 1, 2, and 3 were 77, 80 , and 78, respectively (Table 1 ). Analysis of variance showed no significant difference between treatments, $F(2,44)=0.231, p=.795$ (Table 2). Because no significant difference was found between treatment groups, no further analyses were conducted for the achievement test.

\section{Beliefs}

All subjects completed a 12 -item belief questionnaire. Responses were collected via a seven-point bipolar probability scale. For items 1-10, $7=$ "Strongly Agree" and 1 = "Strongly Disagree;" for item 11, 7 = "Extremely Concerned" and $1=$ "Not at all Concerned;" and for item 12, 7 = "Extremely Often" and $1=$ "Not at All."

There were no significant differences between treatment groups on any of the belief items (Table 3). Subjects in all groups thought the site was credible, with mean responses for item 1 ranging from 5.5-6.1 out of 7. Responses across all groups were also positive for items 2, "I learned a lot from this site," (5.1-5.6) 3, "Learning from this site was easy" (5.5-6.1), and 5, "I thought the site had about

Table 1. Achievement Means and Standard Deviations by Treatment

\begin{tabular}{lccc}
\hline & $\mathrm{T} 1$ & $\mathrm{~T} 2$ & $\mathrm{~T} 3$ \\
\hline Mean score & 77 & 80 & 78 \\
Standard deviation & .1302 & .1256 & .1321 \\
\hline
\end{tabular}

Table 2. Analysis of Variance of Achievement Test

\begin{tabular}{lcccc}
\hline Source & SS & DF & MS & $F$ \\
\hline Between & 7.72 & 2 & 3.86 & $0.231^{*}$ \\
\hline
\end{tabular}

${ }^{*} p=0.795$ 
WHITE SPACE IN LEARNING VIA THE WEB / 197

Table 3. Mean Responses to Belief Questionnaire

\begin{tabular}{|c|c|c|c|c|}
\hline Item & $\mathrm{T} 1$ & $\mathrm{~T} 2$ & Т3 & $\mathrm{F}$ \\
\hline 1. I thought the site was credible. & 5.5 & 5.7 & 6.1 & 1.0 \\
\hline 2. I learned a lot from this site. & 5.6 & 5.3 & 5.1 & 0.8 \\
\hline 3. Learning from this site was easy. & 6.1 & 5.8 & 5.5 & 0.6 \\
\hline $\begin{array}{l}\text { 4. Learning from this site required } \\
\text { concentration. }\end{array}$ & 4.8 & 4.0 & 4.7 & 1.1 \\
\hline $\begin{array}{l}\text { 5. I thought the site had about the right } \\
\text { amount of information. }\end{array}$ & 5.6 & 5.1 & 5.7 & 0.7 \\
\hline $\begin{array}{l}\text { 6. I expect some of my behaviors to } \\
\text { change as a result of visiting this site. }\end{array}$ & 4.5 & 5.1 & 4.2 & 1.5 \\
\hline $\begin{array}{l}\text { 7. In general, Web-based information is } \\
\text { very beneficial. }\end{array}$ & 6.3 & 5.7 & 6.0 & 0.4 \\
\hline $\begin{array}{l}\text { 8. Instructors who use the Internet } \\
\text { are usually better than those who } \\
\text { do not. }\end{array}$ & 4.3 & 4.1 & 4.2 & 0.1 \\
\hline $\begin{array}{l}\text { 9. I trust and respect teachers who } \\
\text { include Internet use in their classes } \\
\text { more than I trust and respect those } \\
\text { who do not. }\end{array}$ & 3.7 & 3.9 & 3.8 & 0.1 \\
\hline $\begin{array}{l}\text { 10. I have had lots of experience with } \\
\text { Web production. }\end{array}$ & 4.9 & 4.5 & 4.1 & 0.8 \\
\hline $\begin{array}{l}\text { 11a. Before visiting the site, I was } \\
\text { concerned about my getting skin } \\
\text { cancer. }\end{array}$ & 3.6 & 3.9 & 2.9 & 1.2 \\
\hline $\begin{array}{l}\text { 11b. Now, after visiting the site, I am } \\
\text { concerned about my getting skin } \\
\text { cancer. }\end{array}$ & 4.7 & 4.2 & 3.8 & 1.5 \\
\hline $\begin{array}{l}\text { 12a. Before I visited the site, I applied } \\
\text { sunscreen this often: }\end{array}$ & 4.3 & 3.8 & 2.4 & 2.7 \\
\hline $\begin{array}{l}\text { 12b. After visiting the site, I expect to } \\
\text { apply sunscreen this often: }\end{array}$ & 4.6 & 4.5 & 3.3 & 1.6 \\
\hline
\end{tabular}


the right amount of information," (5.1-5.7). Responses to question 4, "Learning from this site required concentration" were neutral (4.0-4.7).

For items 6, "I expect some of my behaviors to change as a result of viewing this presentation," and 8, "Instructors who use the Internet are usually better than those who do not," responses were uniformly neutral across treatment groups. Responses to item 7: "In general, Web-based information is very beneficial," ranged from 5.9-6.3. For item 9: "I trust and respect teachers who include Internet use in their classes more than I trust and respect those who do not," response means ranged from 3.8-3.9. Responses to item 10, "I have had lots of experience with Web production," ranged from 4.1-4.9. Responses to items 11 and 12 also did not vary significantly by treatment. Items 11 and 12 did not deal directly with presentation-related issues but focused on individuals' concerns and habits regarding their own health and skin care habits.

\section{DISCUSSION}

The present study measured the effect of specific white space features on learning from instructional Web materials. The study also measured learners' beliefs regarding Web-based instruction. The treatments used in this study were carefully developed to provide sound and accurate information. The overall structure and content were developed carefully and were consistent across all three treatments. The differences between treatments were limited to small differences in a visual component, specifically how white space was handled. Results indicated that in this case, in which the content and overall structure were sound, minor differences with regard to table borders and vertical spacing did not hinder learning.

Hannafin and Hooper [19] have reported that when a learning task is perceived as more difficult, learners may compensate by applying more effort. Reading on-line text certainly demands more concentration than reading printed text. Internet users may compensate by trying harder or concentrating more when reading on-line text. This could work to minimize differences between treatment groups. What seems normal in a given environment also is important. In printed reading, condensed text without structural cues has been shown to decrease comprehension [16]. In the context of printed materials such as a book, poorly presented text as in treatment three would be very unusual and, therefore, noticeable and distracting. However, poor text presentation, including condensed and unstructured text is quite common in the on-line environment. Therefore, in the on-line context, the poor handling of text in treatment three may not have been very noticeable or distracting.

Scrolling may have contributed to a decrease in the positive impact of the control treatment and the lack of scrolling may have contributed to an increase in the appeal of treatment three. Scrolling is sometimes necessary in on-line communication, although most credible Internet development guidelines suggest 
scrolling should be minimized as much as possible [18, 20,21]. While the overall appearance of the control treatment is clearly more appealing and consistent with screen design principles, some scrolling is required. In contrast, treatment three, which is poorest in terms of overall visual appeal and compliance with screen design guidelines, requires little or no scrolling.

Another possible explanation for the finding of no significant difference is learner control, a powerful feature of Web-based instruction. Subjects had unlimited time and were allowed to read the materials as much as they wanted, moving on to more information or returning to earlier information, prior to the posttest. Personal control is an important motivating factor [22-24]. High amounts of learner control is a normal state in on-line learning, as opposed to low or no learner control in preprogrammed computer-generated presentations. Achievement results may have been different if the treatments had precluded learner control, for example, by controlling the amount of time each page was displayed. However, to do so would have been unauthentic, given the nature of the medium. An important attribute of the Web as a medium for learning is that learners generally can control both the amount of time they spend with each screen, and whether or not they return to specific screens for review. For many learners, the Web also may have more intrinsic appeal than ordinary paper-based instructional materials.

Regarding items on the belief questionnaire, the fact that no significant differences were found is not surprising, given the results of the achievement test. On average, subjects in all groups did well on the achievement test, and the content and navigation structures were identical for all treatments.

While it may be tempting to generalize widely and to interpret the results as an indication that white space structural cues and visually appealing layout are not important components of learning from the Web, such is not necessarily the case. All other aspects of the treatments used in the study were carefully developed: content was well structured, clear and concise, and highly credible; text was presented using highly readable font, size, and contrast; navigation was clear and logical; graphics used were high quality, meaningful, and relevant. Generalization should be limited to situations in which other components are carefully designed and developed. Results do not necessarily indicate that white space handling doesn't matter, rather that small differences in white space handling may not be critical when all other aspects are handled well. Follow-up studies should incorporate time on task measures, as well as qualitative techniques such as think aloud sessions, observations, open-ended questions, and interviews to provide deeper understanding of how learners experienced the treatments.

Future research should continue to explore differences in the impact of specific visual aspects of Web instruction. The on-line environment has its own norms, quirks, and rules. For example, underlined and italicized text have important and common uses in printed text. However they should generally be avoided in on-line text as their uses have different meaning or impact on-line. On-line, underlined 
text indicates a clickable link and italicized text is difficult to read (even more so via the Web than in print). Suggested research questions for future related research include the following. Do specific text and layout conventions that research has shown to impact learning in printed instructional materials have similar results when applied to on-line materials? Under what conditions do text and white space related changes hinder or not hinder learning? What are the relationships between intrinsic motivation and cognitive overhead in Web-based instruction? What are the relationships between cognitive overhead and learner control in Web-based

instruction? Research that addresses questions such as these should help define more effective uses of the internet for instructional purposes.

\section{REFERENCES}

1. A. C. Bradshaw, Measuring the Learning Cost of Presentation Interference, in the 20th Annual AECT Proceedings of Selected Research and Development, AECT Publications, Washington, D.C., 1999.

2. A. C. Bradshaw, Evaluating Visuals for Instruction, in Natural Vistas: Visual Literacy and the World Around Us, R. E. Griffin, W. Gibbs, and V. Williams (eds.), International Visual Literacy Association, Loretto, Pennsylvania, 2000.

3. M. Thurling, J. Hanneman, and J. M. Haake, Hypermedia and Cognition: Designing for Comprehension, Communication of the ACM, 38:8, pp. 57-66, 1995.

4. D. Burns, S. Vent, and R. Hansen, The Electronic Publisher, Brady, New York, 1988.

5. J. Felici and T. Nace, Desktop Publishing Skills: A Primer for Typsetting with Computers and Laser Printers, Addison-Wesley, Massachusetts, 1987.

6. T. Lichty, Design Principles for Desktop Publishers, Scott, Foresman, Glenview, Illinois, 1989.

7. J. Hartley, Designing Instructional Text, Kogan Page, London, 1985.

8. M. Munter, Guide to Managerial Communication (3rd Edition), Prentice Hall, Englewood Cliffs, New Jersey, 1992.

9. J. Hartley and D. H. Jonassen, The Role of Headings in Printed and Electronic Text, in The Technology of Text: Principles for Structuring, Designing, and Displaying Text, D. H. Jonassen (ed.), Educational Technology Publications, Englewood Cliffs, New Jersey, pp. 237-263, 1985.

10. B. R. Carosso, Technical Communication, Wadsworth Publishing Co., Belmont, California, 1986.

11. R. Petterson, Visual Information (2nd Edition), Educational Technology Publications, Englewood Cliffs, New Jersey, 1993.

12. A. C. Bradshaw and A. Johari, White Space for Clarity: Suggested Guidelines, in Natural Vistas: Visual Literacy and the World Around Us, R. E. Griffin, W. Gibbs, and V. Williams (eds.), International Visual Literacy Association, Loretto, Pennsylvania, 2000.

13. C. R. Parker, Looking Good in Print, Ventana Press, Chapel Hill, North Carolina, 1988.

14. L. Siebert and L. Ballard, Making a Good Layout, North Light Books, Cincinnati, Ohio, 1992.

15. J. Hartley, Text Design, in Handbook of Research for Educational Communications and Technology, D. Jonassen (ed.), Macmillan, New York, pp. 795-821, 1996. 
16. E. R. Misanchuk, Preparing Instructional Text: Document Design Using Desktop Publishing, Educational Technology Publications, Englewood Cliffs, New Jersey, 1992.

17. J. A. Laspina, The Visual Turn and the Transformation of the Textbook, Lawrence Erlbaum Associates, Mahwah, New Jersey, 1998.

18. J. Nielson, Designing Web Usability, New Riders Publishing, Indianapolis, Indiana, 2000.

19. M. Hannafin and S. Hooper, An Integrated Framework for CBI Screen Design and Layout, Computers in Human Behavior, 5, pp. 155-165, 1989.

20. P. J. Lynch and S. Horton, Web Style Guide: Basic Design Principles for Creating Web Sites, Yale University Press, New Haven, Connecticut, 1999.

21. R. Williams and J. Tollet, The Non-Designer's Web Book: An Easy Guide to Creating, Designing, and Posting your Own Website, Peachpit Press, Berkley, California, 1998.

22. R. deCharms, Personal Causation: The Internal Affective Determinants of Behavior, Academic Press, New York, 1968.

23. E. Deci and R. Ryan, Intrinsic Motivation and Self-Determination in Human Behavior, Plenum, New York, 1985.

24. P. G. Zimbardo, Psychology and Life (14th Edition), HarperCollins, New York, 1996.

Direct reprint requests to:

Dr. Amy C. Bradshaw

Instructional Psychology \& Technology

820 Van Vleet Oval, Collings Hall, 321

University of Oklahoma

Norman, OK 73019 